\title{
Use of antenatal services and delivery care among women in rural western Kenya: a community based survey
}

\author{
Anna M van Eijk ${ }^{* 1,4}$, Hanneke M Bles ${ }^{1}$, Frank Odhiambo², John G Ayisi², \\ Ilse E Blokland ${ }^{1}$, Daniel H Rosen ${ }^{3}$, Kubaje Adazu ${ }^{2}$, Laurence Slutsker ${ }^{4}$ and \\ Kim A Lindblade 4
}

Address: ${ }^{1}$ University of Amsterdam, the Netherlands, ${ }^{2}$ Kenya Medical Research Institute, Centre for Vector Biology and Control Research, Kisumu, Kenya, ${ }^{3}$ Global AIDS Program, Centers for Disease Control and Prevention, Atlanta, GA, USA and ${ }^{4}$ Division of Parasitic Diseases, National Center for Infectious Diseases, Centers for Disease Control and Prevention, Atlanta, GA, USA

Email: Anna M van Eijk* - vaneijka@zimcdc.co.zw; Hanneke M Bles - hannekebles@hotmail.com; Frank Odhiambo - fodhiambo@ke.cdc.gov; John G Ayisi - jayisi@kisian.mimcom.net; Ilse E Blokland - ilseblokland@hotmail.com; Daniel H Rosen - rosend@zimcdc.co.zw;

Kubaje Adazu - kadazu@ke.cdc.gov; Laurence Slutsker - lms5@cdc.gov; Kim A Lindblade - kil2@cdc.gov

* Corresponding author

Published: 06 April 2006

Reproductive Health2006, 3:2 doi:10.1 186/1742-4755-3-2
Received: 08 February 2006

Accepted: 06 April 2006

This article is available from: http://www.reproductive-health-journal.com/content/3/I/2

(C) 2006van Eijk et al; licensee BioMed Central Ltd.

This is an Open Access article distributed under the terms of the Creative Commons Attribution License (http://creativecommons.org/licenses/by/2.0), which permits unrestricted use, distribution, and reproduction in any medium, provided the original work is properly cited.

\begin{abstract}
Background: Improving maternal health is one of the UN Millennium Development Goals. We assessed provision and use of antenatal services and delivery care among women in rural Kenya to determine whether women were receiving appropriate care.
\end{abstract}

Methods: Population-based cross-sectional survey among women who had recently delivered.

Results: Of 635 participants, 90\% visited the antenatal clinic (ANC) at least once during their last pregnancy (median number of visits 4). Most women (64\%) first visited the ANC in the third trimester; a perceived lack of quality in the ANC was associated with a late first ANC visit (Odds ratio [OR] I.5, 95\% confidence interval [CI] I.0-2.4). Women who did not visit an ANC were more likely to have $<8$ years of education (adjusted OR [AOR] 3.0,95\% $\mathrm{Cl}$ I.5-6.0), and a low socio-economic status (SES) (AOR 2.8, 95\% Cl 1.5-5.3). The ANC provision of abdominal palpation, tetanus vaccination and weight measurement were high (>90\%), but provision of other services was low, e.g. malaria prevention $(21 \%)$, iron $(53 \%)$ and folate $(44 \%)$ supplementation, syphilis testing (19.4\%) and health talks (14.4\%). Eighty percent of women delivered outside a health facility; among these, traditional birth attendants assisted $42 \%$, laypersons assisted $36 \%$, while $22 \%$ received no assistance. Factors significantly associated with giving birth outside a health facility included: age $\geq 30$ years, parity $\geq 5$, low SES, $<8$ years of education, and $>$ I hour walking distance from the health facility. Women who delivered unassisted were more likely to be of parity $\geq 5$ (AOR 5.7, 95\% Cl 2.8-II.6).

Conclusion: In this rural area, usage of the ANC was high, but this opportunity to deliver important health services was not fully utilized. Use of professional delivery services was low, and almost I out of 5 women delivered unassisted. There is an urgent need to improve this dangerous situation. 


\section{Background}

In 2000, it was estimated that approximately 529000 women died from complications related to pregnancy or delivery [1]. The majority of maternal deaths occur in developing countries. Causes of maternal deaths include complications of abortion, obstetric complications such as hemorrhage, dystocia, eclampsia, sepsis and infections such as tuberculosis and HIV-1 [2,3]. Attendance at antenatal clinics (ANCs) and receipt of professional delivery care have been associated with a reduction in maternal deaths $[4,5]$. The ANC system in developing countries has been adapted from developed countries without formal evaluations of the impact of interventions in developing country settings. [6]. More recently, studies have been conducted to identify procedures proven to improve reproductive health [7-9]. These evaluations demonstrated that in low risk pregnancies (pregnancies with no abnormal obstetric or medical history), a decrease from 12 ANC visits, as had been previously recommended, to a less-costly 4 visit-schedule did not result in an increase in adverse maternal and perinatal events [10-12]. As a consequence, the World Health Organization now recommends a 4-visit ANC schedule for low risk pregnancies [13]. Other interventions shown to be beneficial to mother and child include routine iron and folate supplementation in areas with a high prevalence of anemia, serologic screening for and treatment of syphilis, routine measurement of fundal height, malaria prevention, and tetanus immunization [9]. To fully benefit from these interventions, it is important that women begin attending ANC early in pregnancy.

In many developing countries, the majority of births occur without the help of a skilled assistant (defined as a midwife, nurse trained as midwife, or a doctor) at home or in other non-hospital settings [14]. Home deliveries in the absence of skilled professional attendants have been associated with adverse infant and maternal outcome $[15,16]$. However, home deliveries without a skilled attendant are chosen or occur for a variety of reasons, including long distances or difficult access to a birth facility, costs of services and perceived lack of quality of care in a health facility [17-19]. In an attempt to improve care during home deliveries and reduce maternal mortality, traditional birth attendants (TBAs) have been trained in modern delivery care, with varying reports of success [2022]. Presence of a professional attendant at each birth can lead to a marked reduction in maternal mortality and morbidity $[15,16]$; professional health care during childbirth is one of the process indicators to assess progress towards the Millennium Development Goal of improving maternal health [23].

We conducted a community-based survey in rural western Kenya, where anemia, malaria and HIV rates are high, among a representative sample of women who had recently delivered, to identify the locations where they had sought care during their pregnancy and delivery and the range of services they had received. This information will help to improve maternal health care in this area.

\section{Methods}

The survey was conducted in the rural area of Asembo (Rarieda Division) and Gem (Wagai and Yala Divisions) in western Kenya. Asembo has a population of approximately 55,000 people living in 79 villages. Gem has 75,000 inhabitants living in 144 villages. This area historically experienced intense perennial malaria transmission before the initiation of a large community-based trial of insecticide treated bed nets (ITNs) that reduced malaria transmission considerably [24,25]. Following this trial, a health and demographic surveillance system (HDSS) was established in the same area [26]. All births, deaths, pregnancies and in- and out migrations are recorded and updated every four months. Socio-economic information and educational level are obtained and updated annually. All households who participate in the HDSS receive ITNs; free retreatment is available in each village every 9 months.

Using a population-based sampling frame of women in the HDSS who had recently delivered, we selected a random sample of 730 women. We estimated that a sample size of 730 was required to calculate the proportion of those using antenatal or delivery care in both Asembo and Gem within 5 percentage points of the true proportion, assuming the true proportion was $70 \%$ and that $12 \%$ of women would not be available. Trained interviewers visited the selected women at home and administered a standardized questionnaire in the local language. Participants were asked if they had visited an ANC during their last pregnancy. If they answered "Yes," they were asked to specify their reasons for doing so; this question was repeated until no new reasons were reported. Answers were tick marked in predefined options or described under "other reasons." Interviewers were instructed not to probe with options. Socio-economic information was obtained from the HDSS. Information on household assets was used to derive a wealth index using the method developed by Filmer \& Pritchet (2000) [27]. All households were categorized by quintile: a medium/low socioeconomic status (SES) was defined as an SES in the bottom 3 quintiles of the wealth index.

Differences in proportions were compared using the Chisquare test or Fisher's exact test when appropriate, and differences in means were compared using the Student's $t$ test (SPSS for Windows 11.0, SPSS Inc. Chicago, Illinois). A two-sided $P$-value $<0.05$ was considered statistically significant. Odds ratios with $95 \%$ confidence intervals were 
calculated. Results were stratified by parity, age, level of education ( $<8$ years versus $\geq 8$ years of education) area of residence (Asembo versus Gem), and history of child death or previous stillbirth. Infrequent usage of ANC was defined as having made $<3$ ANC visits, the minimum number needed to benefit from intermittent preventive treatment with sulfadoxine-pyrimethamine for malaria. Primiparae were women who had delivered for the first time, multiparae were women who had delivered more than once. Grande multiparae were defined as women who had delivered 5 or more times, given that a high number of previous deliveries is an obstetric risk factor. Logistic regression was used to assess the effect of various factors on ANC attendance, place of delivery and type of attendant at delivery. The effect of the following factors was examined: maternal age, parity, marital status, socioeconomic status, education level, area of residence (Gem versus Asembo), distance to the ANC and history of stillbirth or child death. Factors significant in the univariate analysis were included in the multivariate analysis.

The project was reviewed and approved by the institutional review boards of the Centers for Disease Control and Prevention (Atlanta, GA) and the Kenya Medical Research Institute (Nairobi, Kenya). All women who participated gave written informed consent after reading through the consent form with the interviewer; participants who could not write indicated their consent by a fingerprint, which was witnessed by the interviewer.

\section{Results}

\section{Study population}

Interviews were conducted in December 2002. Of the 730 women who were selected, 8 were found not to have recently delivered and were excluded. Eighty-two (11.4\%) of the remaining 722 eligible women could not be reached during the period of survey. An additional 3 women $(0.4 \%)$ had died in the interval between delivery and interview and information from 2 women $(0.3 \%)$ was assessed to be unreliable. The 87 women (median age 22 years) who could not be interviewed (12\% of the eligible sample) were significantly younger than the 635 women included in the analysis (median age 25 years, $P<$ 0.001 , Mann-Whitney U test). The time interval between interview and last delivery was less than 6 months for $89 \%$ of the interviews and the maximum interval was 11 months. The number of years of education, socio-economic status, and time interval between interview and delivery were similar between women interviewed and those not interviewed (number of years of education 6.8 versus 6.4 years, respectively, $P=0.5$; a low/medium SES $57 \%$ versus $57 \%$, respectively, $P=1.0$; interval between delivery and interview: 4.0 versus 4.2 months, respectively, $P=0.8$, Mann-Whitney U test).
Of the 635 women enrolled, 98\% were members of the Luo ethnic group. The mean age (standard deviation, SD) was 26.5 (6.9) years; 40 women $(6 \%)$ were less than 18 years of age. The median number of pregnancies was 4 (range 1-15). Of the 621 singleton deliveries, 15 (2\%) were stillbirths and by the time of the interview 27 live born infants (4\%) were reported to have died.

\section{Care during pregnancy}

A total of 571 women (90\%) visited an ANC at least once; 34 different ANCs in or around the area of the survey were visited. The number of antenatal visits during pregnancy ranged from 1 to 10 , with a median of 4 . One or two visits only were made by $46(8 \%)$ and $69(12 \%)$ of the women respectively. Among the 559 ANC attendees who could recall when they first attended, 78 women (14\%) started ANC visits in the first trimester, 355 women (64\%) started in the second trimester, and 126 women $(23 \%)$ in the third trimester.

Most (87\%) women decided for themselves to visit the ANC; the husband, mother or mother-in-law suggested attending the ANC for only a few $(5 \%, 4 \%$, and $2 \%$ respectively). Young women and primiparae were more likely to have been advised to attend by their mothers or mothers-in-law than were older or multiparae women (e.g. among young women, $9 \%$ were advised to attend by her mother-in-law versus $1 \%$ among older women, OR 11.0, 95\% CI 3.4-35.9). Many women (67\%) gave more than 1 reason to visit an ANC; the reasons most frequently mentioned were: to check the position, condition or growth of the baby (83\%); to detect maternal problems and to be treated when sick (55\%); to get a tetanus injection (24\%); and to get an ANC card (18\%). Participants expressed the belief that medical staff in health facilities treat pregnant women better if they attend with an ANC card, particularly if the card shows evidence of multiple visits. Eleven percent of the women mentioned that they appreciated the health information the ANC provided in the form of talks or posters.

Most women (93\%) reported walking to the ANC; walking times ranged from 1 minute to 3 hours (median: 40 minutes). Although distance was cited as a barrier to ANC use, 102 women (18\%) did not visit the nearest ANC. Major reasons given for attending a more distant ANC included better perceived care $(78 \%)$, or lower cost $(13 \%)$. Seven percent of women visited more than one ANC, usually because of a temporary move or because services were thought to be better in the second ANC. In addition, $18 \%$ of women sought other sources of care during their pregnancies, such as TBAs $(65 \%)$, religious leaders $(14 \%)$, herbalists $(13 \%)$, or traditional healers $(8 \%)$. 
Table I: Association between maternal characteristics and not visiting an ANC, Asembo/Gem, Western Kenya, December 2002

\begin{tabular}{|c|c|c|c|c|}
\hline & $\begin{array}{l}n\left(\%^{*}\right) \text { who visited an } \\
\text { ANC }(N=57 I)\end{array}$ & $\begin{array}{l}\mathrm{N}\left(\%^{*}\right) \text { who did not visit } \\
\text { an ANC }(\mathrm{N}=64)\end{array}$ & $\begin{array}{l}\text { Factors associated with not } \\
\text { visiting an ANC, } \\
\text { univariate analysis OR }(95 \% \mathrm{Cl})\end{array}$ & $\begin{array}{l}\text { Factors associated with not } \\
\text { visiting an ANC, } \\
\text { multivariable analysis AOR } \\
(95 \% \mathrm{Cl})\end{array}$ \\
\hline \multicolumn{5}{|l|}{ Age } \\
\hline$<18$ years & $34(6.0)$ & $6(9.4)$ & $1.72(0.67-4.43)$ & $1.55(0.36-6.72)$ \\
\hline $18-19$ years & $79(13.8)$ & I (I.6) & $0.12(0.02-0.92)$ & $0.16(0.02-1.27)$ \\
\hline $20-29$ years & $282(49.4)$ & $29(45.3)$ & Reference & Reference \\
\hline $30-34$ years & $99(17.3)$ & $9(14.1)$ & $0.88(0.40-1.93)$ & $0.53(0.21-1.32)$ \\
\hline$>34$ years & 77 (13.5) & $19(29.7)$ & $2.40(1.28-4.5 I)$ & $1.16(0.5 \mathrm{I}-2.67)$ \\
\hline \multicolumn{5}{|l|}{ Parity } \\
\hline Para I & $103(18.0)$ & $8(12.5)$ & $1.20(0.49-2.91)$ & $1.25(0.33-4.80)$ \\
\hline Para 2-4 & $231(40.5)$ & $15(23.4)$ & Reference & Reference \\
\hline Para $\geq 5$ & $237(4 I .5)$ & $4 I(64.1)$ & $2.66(1.44-4.95)$ & $1.95(0.85-4.49)$ \\
\hline \multicolumn{5}{|l|}{ Marital status } \\
\hline Single/widow & $79(13.9)$ & $9(14.1)$ & $1.02(0.48-2.14)$ & \\
\hline Married & $491(86.1)$ & $55(85.9)$ & Reference & \\
\hline \multicolumn{5}{|l|}{ Socio-economic status } \\
\hline Low/medium & $303(54.5)$ & $49(76.6)$ & $2.73(1.49-4.98)$ & $2.82(1.49-5.34)$ \\
\hline High & $253(45.5)$ & $15(23.4)$ & Reference & Reference \\
\hline \multicolumn{5}{|l|}{ Education level } \\
\hline$<8$ years & 291 (52.6) & $51(81.0)$ & $3.83(2.00-7.33)$ & $3.02(1.51-6.04)$ \\
\hline$\geq 8$ years & $262(47.4)$ & $12(19.0)$ & Reference & Reference \\
\hline \multicolumn{5}{|l|}{ Residence } \\
\hline Asembo & $293(51.3)$ & $26(40.6)$ & $0.65(0.38-1.10)$ & \\
\hline Gem & $278(48.7)$ & $38(59.4)$ & Reference & \\
\hline \multicolumn{5}{|l|}{ Distance to ANC } \\
\hline Walking $<$ I hr & $298(52.2)$ & $27(42.2)$ & Reference & Reference \\
\hline Walking I hr & $128(22.94)$ & $16(25.0)$ & $1.38(0.72-2.65)$ & I.I3 (0.57-2.23) \\
\hline Walking > I hr & $107(18.7)$ & $20(31.3)$ & $2.06(1.11-3.83)$ & $1.55(0.80-3.03)$ \\
\hline Used bus or bike & $38(6.7)$ & I (I.6) & $0.29(0.04-2.20)$ & $0.40(0.05-3.12)$ \\
\hline \multicolumn{5}{|l|}{ Previous child-death } \\
\hline$\geq 3$ times & $38(6.7)$ & $9(14.1)$ & $2.79(|.23-6.3|)$ & $1.40(0.52-3.74)$ \\
\hline I or 2 times & $180(31.5)$ & $25(39.1)$ & $1.63(0.93-2.86)$ & $1.14(0.59-2.17)$ \\
\hline None & $353(61.8)$ & $30(46.9)$ & Reference & Reference \\
\hline \multicolumn{5}{|l|}{ Previous stillbirth } \\
\hline Yes & $65(11.4)$ & $8(12.5)$ & I.II (0.5I-2.44) & \\
\hline No & $506(88.6)$ & $56(87.5)$ & Reference & \\
\hline
\end{tabular}

Abbreviations: OR, odds ratio; AOR, adjusted odds ratio; $\mathrm{Cl}$, confidence interval; hr:hour. Significant (adjusted) odds ratios printed in bold. *Column percentages

Incomplete and inadequate services at the ANC visited were complaints mentioned by $29 \%$ of women who visited an ANC. These women were more likely to start ANC in the third trimester (28\% versus $20 \%$ among women who did not complain about the quality of ANC services, OR $1.5,95 \%$ CI $1.0-2.3, P=0.05)$, and had a lower median number of ANC visits (3), although this was not statistically significant (Mann-Whitney U test, $P=0.1$ ).

A total of 64 women (10\%) never attended an ANC during their most recent pregnancy. The most frequently mentioned reasons for not attending were not seeing the need to attend (36\%), expenses of transport or the cost of the ANC (27\%), belief that the care was not adequate $(22 \%)$, and distance to the ANC (14\%). Of the 64 who did not attend, $27 \%$ sought alternative care during pregnancy from sources such as TBAs, religious persons, or herbalists.

Factors associated with not attending an ANC are summarized in table 1 . Adolescents (women $<18$ years) and older women ( $>34$ years) were the least likely to attend. In a multivariate model, only $<8$ years of education (adjusted odds ratio [AOR] 3.0, 95\% CI 1.5-6.0) and medium/low SES (AOR 2.8, 95\% CI 1.5-5.3) remained associated with never attending an ANC.

Factors associated with infrequent visits ( $<3$ times) among women who visited the ANC were similar to factors associated with not visiting at all, with the addition 
Table 2: Services offered in the ANCs visited during the last pregnancy, Asembo/Gem, Western Kenya, December 2002*

\begin{tabular}{ll}
\hline Services offered in ANC & $\mathrm{n}(\%)$ \\
\hline Palpation of the abdomen & $562(98.4)$ \\
Tetanus vaccination & $555(97.2)$ \\
Weight measurement & $518(90.7)$ \\
Blood pressure measurement & $385(67.4)$ \\
Iron supplementation & $303(53.1)$ \\
Hemoglobin measurement & $281(49.2)$ \\
Folic acid supplementation & $253(44.3)$ \\
SP $\geq$ I dose) & $121(21.2)$ \\
Syphilis test & $111(19.4)$ \\
Urine test & $89(15.6)$ \\
Information on danger signs during pregnancy & $82(14.4)$ \\
Health talk & $80(14.0)$ \\
Stool test & $72(12.6)$ \\
Antihelmintics & $15(2.6)$
\end{tabular}

Abbreviations: ANC: antenatal clinic; SP: sulfadoxine-pyrimethamine *Note: no information was obtained in the questionnaire on height measurement, or dose and duration of iron and folic acid supplementation, or HIV-counseling and testing. The information was not checked with an ANC card/book if available.

that being a single woman (single, separated, or divorced: AOR 2.5, 95\% CI 1.3-5.0) was associated with making $<3$ visits to the ANC.

\section{Services offered by the ANC}

Almost all women were examined and received a tetanus vaccination during their ANC-visits (Table 2). However, other preventive treatments, laboratory tests, and health education were not common, ranging from $67 \%$ for a blood pressure measurement to $3 \%$ for treatment for helminthiasis. One out of 5 women received malaria prevention $(21 \%)$ or underwent a syphilis test (19\%), and approximately half of the women received iron (53\%) and folate (44\%) supplementation. Among the $80(14 \%)$ women who received health talks during ANC visits, the two most frequent topics were care during pregnancy (recommended diet, avoiding a heavy workload, importance of regular ANC attendance and a hospital delivery), and care for the newborn (e.g. diet, breastfeeding). Family planning (mentioned by 5 women), the use of bed nets to prevent malaria (3), and HIV prevention (3) were infrequent health education topics.

Tetanus toxoid injections are given during pregnancy to prevent neonatal tetanus, a frequent cause of infant deaths when sterile procedures are not observed in cutting the umbilical cord following delivery. Women in their first pregnancy typically receive 2 doses (TT1-TT2) with 1 month in between; subsequent doses are recommended after a minimum of half a year (TT3) or one year apart (TT4-TT5), and are in general given during ANC visits for subsequent pregnancies $[13,28]$. Five doses are consid- ered to provide life-long immunity. Among 570 women who could recall the number of tetanus doses received, $97 \%$ received at least one dose; $46 \%$ received only one dose, $43 \%$ received 2 doses, and $8 \%$ received $\geq 3$ doses. Although any coverage with tetanus vaccination was generally high, among primiparae $29 \%$ reported one dose only, whereas $41 \%$ of para $\geq 5$ reported $\geq 2$ doses.

\section{Care during delivery}

Most women (83\%) delivered outside of a health facility. Of these, $80 \%$ delivered in their own house, $18 \%$ in the house of a TBA and 3\% on their way to a health facility. The most frequent reason for not attending a health facility for delivery was lack of means of transport, in particular at night $(49 \%)$. Other important barriers were fast progression of labor (47\%), and expense (28\%). Fourteen percent of women did not think facility attendance was necessary; reasons given for this included previous uneventful home delivery, preferred home deliveries, or had made arrangements with TBAs or another person to attend the delivery. A small subset (3\%) reported anticipation of unpleasant treatment at a health facility as a reason not to attend. Of note, $64 \%$ of those who delivered outside a health facility were aware of the potential risks, and could identify one or more complications that could occur. Among women who did not visit an antenatal clinic, only 1 woman (1.6\%) delivered in a health facility; this was $10.2 \%$ among the 266 women who made $1-3$ visits, and $27.2 \%$ among the 305 women who made 4 or more visits (Trend test $P<0.001$ ). In multivariate analysis, factors associated with delivery outside a health facility included: age $\geq 30$ years, parity $\geq 5$, low/medium SES, $<8$ years of education, and $>1$ hour walking distance from the hospital (Table 3).

Among all women, only $17 \%$ were attended to by a professionally trained provider (doctor, nurse, midwife, or clinical officer) and TBAs assisted another 36\% of women. A disturbingly high proportion of women (29\%) were attended to by an untrained family member, friend or neighbor, and $18 \%$ of women delivered completely unattended (Figure 2). In multivariate analysis, women who delivered on their own were more likely to be of parity $\geq$ 5 (AOR 5.7, 95\% CI 2.8-11.6), whereas women who had lost a child previously (any child death) were less likely to deliver on their own (AOR 0.5, 95\% CI 0.3-0.9). Age, education level, SES, marital status, and distance to ANC were not significant factors in this model (data not shown).

\section{Discussion}

In this survey in a rural area in western Kenya, 9 out of 10 women reported at least one ANC visit during their last pregnancy; however, two-thirds of these women began attending the ANC in the third trimester, and only half of 
these women made the recommended number of 4 visits. Services provided by the various ANCs were not optimal, with a low coverage of intermittent preventive treatment with sulfadoxine-pyrimethamine, and supplementation of iron and folic acid. The proportion of women assisted during delivery by a professionally trained provider $(17 \%)$ was similar to the proportion of women who delivered on their own (18\%). Untrained persons and TBAs assisted in the majority of deliveries (65\%).

The high ANC attendance (90\%) in this rural area is encouraging, and is similar to a report for the whole of Kenya (88\%) [29]. Having more than 8 years of education and being of higher socio-economic status were the most important factors associated with ANC attendance; these findings are similar to those from an evaluation of antenatal care attendance in developing countries [13]. Although the perceived expense of the ANC may hinder attendance, it is uncertain that free antenatal care would increase coverage substantially because transport costs, physical inability to travel long distances, and a perceived poor quality of care would remain barriers. Unlike Nigeria and Uganda, where the husband played an important role in determining their partner's ANC attendance, in our study most women made an independent decision to attend $[17,30]$.

There were fewer services offered by the ANCs attended by the women in this study compared to the whole of Nyanza Province; participants in this survey were half as likely to receive information on danger signs in pregnancy, or to receive a urine test (30\% versus $14 \%$, and $35 \%$ versus $16 \%$ respectively) than participants in the 2003 Demographic and Health Survey (DHS), and they were less likely to report a blood pressure measurement (67\% versus $76 \%, P=0.001$ ) [29]. Women appreciated the information and advice received at the ANC. The main topics were care during pregnancy and care for the newborn. However, few women attended a health talk (14\%), and other essential topics such as place of delivery, making an individual birth plan, family planning, malaria, and HIV/AIDS prevention received little attention. Information on the effect of health education on maternal and perinatal outcome in sub-Saharan Africa is limited; a study in Tanzania assessing the effect of group and individual counseling on the prevention of anemia did not see an additional effect of this approach above the intervention of ensuring an adequate supply of hematinics and malaria prophylaxis [31]. Other services with proven benefit had a low coverage in this survey as well; barely half of the women received hematinic supplements, one out of 5 women received one or more doses of sulfadoxinepyrimethamine for malaria, and an antihelmintic treatment was received by $3 \%$. This is in stark contrast with the needs as confirmed during a cross-sectional survey in July
2003 among 673 pregnant women in the same area; 36\% of the women were malaria parasitemic $(52 \%$ among primigravidae), 53\% were anemic (hemoglobin $<11 \mathrm{~g} / \mathrm{dl}$ ) and $76 \%$ of the 391 women who had brought a stool sample were infected with a nematode (van Eijk et al., unpublished). In our study, we did not investigate the reasons why many ANC visits passed without provision of these services, e.g. if there was a lack of supply of drugs; this is a focus of future evaluations.

Consistent with a report from a neighboring area, only $37 \%$ of the woman started attending ANC in the first or second trimester [32]. Low ANC attendance in the first trimesters has also been reported in other African and developing countries $[19,29,30,33,34]$. Late ANC attendance may preclude women from benefiting fully from preventive strategies, such as iron and folic acid supplementation, treatment of helminthic infections, and intermittent preventive treatment with sulfadoxine-pyrimethamine for malaria in pregnancy. Late attendance was associated with a perceived lack of services at the ANC. As summarized in table 2, provision of services in this area was generally poor and worse than that reported in the national ANC service profile in the most recent DHS [29]. To encourage earlier ANC attendance, service delivery must be improved.

Despite the fact that $90 \%$ of the women reported attending antenatal care, fewer than 2 in 10 gave birth in a health facility. This is lower than the national estimate of $40 \%$ for Kenya and 39\% for Nyanza Province [29], although higher than that reported in some other African countries [14]. Observed contributing factors for a home delivery included the fast progression of labor, distance, difficulty of (night) travel, and cost. Distance was a barrier for facility delivery but not for ANC attendance. This may be because not every ANC facility has 24-hour maternity service, so the distance to reach a health facility with maternity service may be greater than for antenatal care. In addition, given the urgent nature of deliveries, there may be less time to cover the distance. Other work has confirmed the importance of distance on access to maternity care $[18,34]$.

We were surprised at the high proportion of women who gave birth without any assistance. Our estimate of $18 \%$ was nearly twice that observed in the most recent Kenya DHS (National estimate $8.0 \%$, Nyanza Province 9.8\%) and in a study in Mbeere District, Kenya $(6.5 \%)[19,29]$. Unassisted delivery was particularly common among grande multiparae, a high-risk group in obstetrics $[19,29]$. The lack of any attendant makes it difficult to seek assistance in the event of life-threatening complications. Women should be strongly encouraged to deliver with 
Table 3: Association between maternal characteristics and a birth outside a health facility, Asembo/Gem, Western Kenya, December 2002*

\begin{tabular}{|c|c|c|c|c|}
\hline & $\begin{array}{l}\mathrm{n}(\% *) \text { who delivered in a } \\
\text { health facility }(\mathrm{N}=1 \mathrm{II})\end{array}$ & $\begin{array}{l}\mathrm{n}\left(\%^{*}\right) \text { who delivered } \\
\text { outside a health facility } \\
(\mathrm{N}=524)\end{array}$ & $\begin{array}{l}\text { Factors associated with } \\
\text { birth outside a health } \\
\text { facility, univariate analysis, } \\
\text { OR }(95 \% \mathrm{Cl})\end{array}$ & $\begin{array}{l}\text { Factors associated with birth } \\
\text { outside a health facility, } \\
\text { multivariable analysis, AOR } \\
(95 \% \mathrm{Cl})\end{array}$ \\
\hline \multicolumn{5}{|l|}{ Age } \\
\hline$<18$ years & $10(9.0)$ & $30(5.7)$ & $0.78(0.36-1.67)$ & $0.77(0.28-2.13)$ \\
\hline $18-19$ years & $19(17.1)$ & $61(11.6)$ & $0.83(0.46-1.49)$ & $0.90(0.45-1.82)$ \\
\hline $20-29$ years & $64(57.7)$ & $247(47.1)$ & Reference & Reference \\
\hline $30-34$ years & $10(9.0)$ & $98(18.7)$ & $2.54(1.25-5.15)$ & $1.65(0.69-3.94)$ \\
\hline$>34$ years & $8(7.2)$ & 88 (91.7) & $2.85(1.31-6.18)$ & $1.25(0.48-3.24)$ \\
\hline \multicolumn{5}{|l|}{ Parity } \\
\hline Para I & $30(27.0)$ & $81(15.5)$ & $0.80(0.48-1.33)$ & $0.98(0.47-2.05)$ \\
\hline Para 2-4 & $56(50.5)$ & $190(36.3)$ & Reference & Reference \\
\hline Para $\geq 5$ & $25(22.5)$ & $253(48.3)$ & $2.98(1.80-4.96)$ & $\mathrm{I} .63(0.8 \mathrm{I}-3.26)$ \\
\hline \multicolumn{5}{|l|}{ Marital status } \\
\hline Single/Widow & $22(19.8)$ & $66(12.6)$ & $0.58(0.34-0.99)$ & $0.90(0.45-1.8 I)$ \\
\hline Married & $89(80.2)$ & $457(87.4)$ & Reference & Reference \\
\hline \multicolumn{5}{|l|}{ Socio-economic status } \\
\hline Low/medium SES & $49(46.7)$ & $303(58.8)$ & $1.63(1.07-2.49)$ & $1.6 \mathrm{I}(1.02-2.54) \dagger$ \\
\hline High SES & $56(53.3)$ & $2 \mid 2(4 \mid .2)$ & Reference & Reference \\
\hline \multicolumn{5}{|l|}{ Education level } \\
\hline$<8$ years & $44(41.5)$ & $298(58.4)$ & $1.98(1.30-3.03)$ & $1.68(1.04-2.69) \dagger$ \\
\hline$\geq 8$ years & $62(58.5)$ & $212(4 \mid .6)$ & Reference & Reference \\
\hline \multicolumn{5}{|l|}{ Residence } \\
\hline Asembo & $60(54.1)$ & $259(49.4)$ & $0.83(0.55-1.25)$ & \\
\hline Gem & $51(45.9)$ & $265(50.6)$ & Reference & \\
\hline \multicolumn{5}{|l|}{ Travel to ANC } \\
\hline Walking < I hour & $74(66.7)$ & $25 I(47.9)$ & Reference & Reference \\
\hline Walking I hour & $18(16.2)$ & $126(24.0)$ & $2.06(1.18-3.61)$ & $1.71(0.95-3.07)$ \\
\hline Walking > I hour & $10(9.0)$ & $117(22.3)$ & $3.45(1.72-6.92)$ & $2.75(1.33-5.68)$ \\
\hline Used bus or bicycle & $9(8.1)$ & $30(5.7)$ & $0.98(0.45-2.16)$ & $1.24(0.50-3.07)$ \\
\hline \multicolumn{5}{|l|}{ Previous child-death } \\
\hline$\geq 3$ times & $3(2.7)$ & $44(8.4)$ & $4.18(1.27-13.81)$ & $1.35(0.36-5.10)$ \\
\hline I or 2 times & $23(20.7)$ & $182(34.7)$ & $2.26(1.37-3.7 I)$ & $1.60(0.89-2.88)$ \\
\hline None & $85(76.6)$ & $298(56.9)$ & Reference & Reference \\
\hline \multicolumn{5}{|l|}{ Previous stillbirth } \\
\hline Yes & $14(12.6)$ & $59(11.3)$ & $0.88(0.47-1.64)$ & \\
\hline No & $97(87.4)$ & $465(88.7)$ & Reference & \\
\hline
\end{tabular}

Abbreviations: OR, odds ratio; AOR, adjusted odds ratio; $\mathrm{Cl}$, confidence interval; SES: socio-economic status. Significant odds ratios printed in bold. *Column percentages

†The introduction of number of ANC visits ( $\geq 4$ visits, I-3 visits vs. no visit as reference) in the multivariate model reduced the AOR of Low/ medium SES (AOR I.36, 95\% Cl 0.85-2.17), and education level < 8 years (AOR I.46, 95\% Cl 0.90-2.38), but did not affect the other variables in the model. The AOR for $\geq 4$ visits was 15.08 (95\% Cl I.99-II4), and for I-3 visits was $2.8 \mathrm{I}(\mathrm{I} .68-4.69)$.

assistance. The promotion of a delivery plan may be a good step towards sensitizing women on this issue.

TBAs frequently form the backbone of maternity services in rural areas, and in our survey they attended about onethird of deliveries. It has been suggested that training of TBAs could reduce maternal and perinatal mortality, but recent data have not supported this strategy $[20,35,36]$. Some participants preferred TBAs because of greater flexibility in payment.
Limitations of this survey include the fact that the sample of women who could not be found were younger than those enrolled, and young women were more likely to visit the ANC; however, even if all excluded women would have visited the ANC, the estimate of the prevalence of having attended ANC at least once would not have changed substantially. We did not cross check the information provided by the women during the interviews with ANC card data; thus, services actually provided could have been underreported. Lastly, only women who sur- 


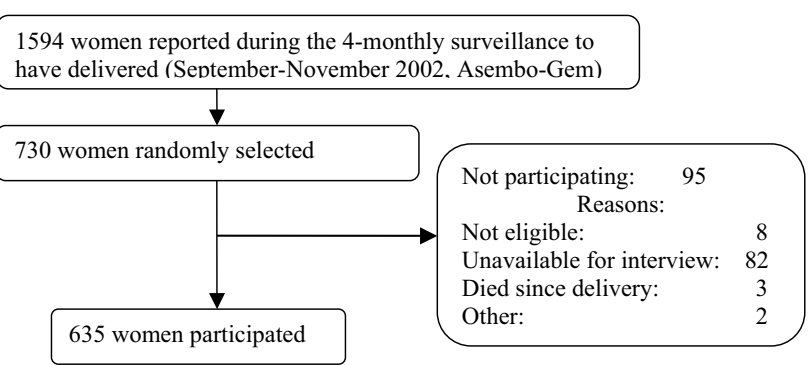

Figure I

Flow chart of the survey population, Asembo/Gem, Western Kenya, December 2002.

vived their last delivery were able to participate in this survey.

\section{Conclusion}

The Safe Motherhood Initiative promotes antenatal care, and skilled assistance (defined as a midwife, nurse trained as midwife, or a doctor) during childbirth [37,38]. Based on this definition, there is much to be done to achieve 'Safe Motherhood' in rural areas of western Kenya: a trained provider attended fewer than 1 in 5 deliveries. The discrepancy between the national and provincial estimates of unassisted deliveries or deliveries without skilled assistance and our findings illustrate that these estimates may underestimate the situation in the rural areas. Safe motherhood strategies promote counseling of clients on danger signs and the development of an individual birth plan; pregnant women and their relatives are encouraged to arrange transport, money and a companion before onset of labor [28]. This survey indicates that the situation in western Kenya needs urgent improvement. The Kenya Ministry of Health is working on expanding focused antenatal care and prevention of malaria in pregnancy in rural areas [28]. This survey can provide baseline data for this rural area against which efforts to improve services in the ANCs can be evaluated.

\section{Competing interests}

The author(s) declare that they have no competing interests.

\section{Authors' contributions}

Amve was involved in the design of the study, in analysis and interpretation of the data, and in drafting the paper. $\mathrm{HB}$ and IEB were involved in the analysis and interpretation of the data and in drafting the paper. FO, JGA and KA were involved in data collection, and revision of the manuscript. DHR was involved in statistical analysis and interpretation of the data, and revising the manuscript. LS was involved in design, interpretation of the data and review of the manuscript. KAL was involved in conception and

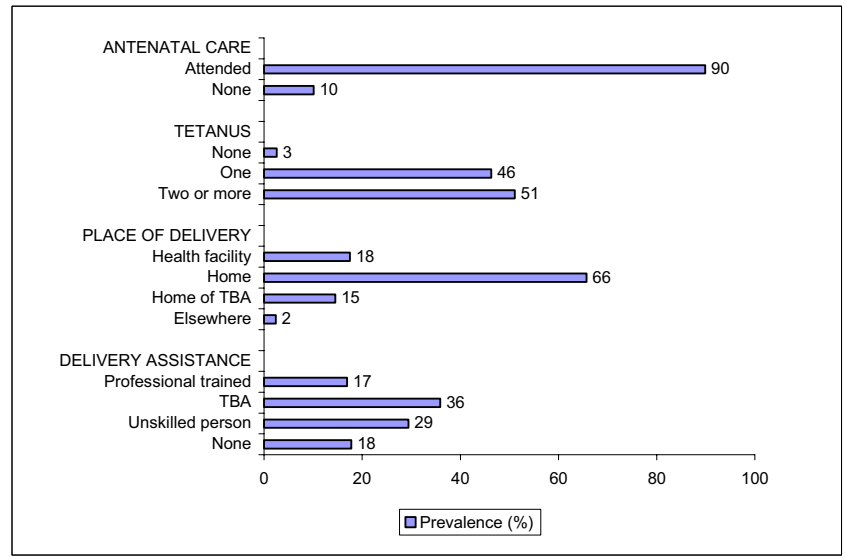

Figure 2

ANC attendance, number of tetanus doses, place of delivery and attendants, Asembo/Gem, Western Kenya, December 2002.

design, interpretation of the data and drafting and reviewing the manuscript. Each author has given final approval of the version to be published.

\section{Acknowledgements}

We thank the participants in Asembo and Gem for their time and willingness to share their experiences. We are grateful for the dedicated performance of the field and data management staff of the HDSS. We thank the $\mathrm{KEMRI}$ and CDC/KEMRI administrative staff for the support they provided and continue to provide to the project. This survey was sponsored by CDC. Dr. A. van Eijk was supported by the Oak Ridge Institute for Science and Education, Tennessee, USA. This paper was published with the approval of the Director of the Kenya Medical Research Institute.

\section{References}

I. World Health Organization, UNICEF, UNFPA: Maternal mortality in 2000: estimates developed by WHO, UNICEF and UNFPA. Geneva, Switzerland; 2004.

2. Inter-Agency Group for Safe Motherhood: The safe motherhood action agenda: priorities for the next decade. Report on the safe motherhood technical consultation 18-23 October 1997. Colombo, Sri Lanka; 1997.

3. Khan M, Pillay T, Moodley JM, Connolly CA: Maternal mortality associated with tuberculosis-HIV-I co-infection in Durban, South Africa. AIDS 200I, I5:1857-1863.

4. Magadi M, Madise N, Diamond I: Factors associated with unfavourable birth outcomes in Kenya. J Biosoc Sci 200I, 33: 199-225.

5. UNICEF Eastern and Southern Africa Regional Office: Maternal Mortality Reduction Strategy. 2003.

6. World Health Organization, UNICEF: Antenatal care in developing countries. Promises, achievements and missed opportunities. An analysis of trends, levels and differentials, 1990200 I. Geneva, Switzerland; 2003.

7. Villar J, Bergsjo P: Scientific basis for the content of routine antenatal care. I. Philosophy, recent studies, and power to eliminate or alleviate adverse maternal outcomes. Acta Obstet Gynecol Scand 1997, 76: I- I4.

8. Bergsjo P, Villar J: Scientific basis for the content of routine antenatal care. II. Power to eliminate or alleviate adverse newborn outcomes; some special conditions and examinations. Acta Obstet Gynecol Scand 1997, 76:15-25.

9. Lumbiganon P: Appropriate technology: antenatal care. Int J Gynaecol Obstet 1998, 63(SuppI I):S9I-S95. 
10. Pittrof $R$, Campbell OM, Filippi VG: What is quality in maternity care? An international perspective. Acta Obstet Gynecol Scand 2002, $81: 277-283$.

II. Carroli G, Villar J, Piaggio G, Khan-Neelofur D, Gulmezoglu M, Mugford M, Lumbiganon P, Farnot U, Bersgjo P: WHO systematic review of randomised controlled trials of routine antenatal care. Lancet 200I, 357:I565-I570.

12. Villar J, Ba'aqeel H, Piaggio G, Lumbiganon P, Miguel BJ, Farnot U, AlMazrou Y, Carroli G, Pinol A, Donner A, Langer A, Nigenda G, Mugford M, Fox-Rushby J, Hutton G, Bergsjo P, Bakketeig L, Berendes H, Garcia J: WHO antenatal care randomised trial for the evaluation of a new model of routine antenatal care. Lancet 200I, 357: I55I-I564.

13. World Health Organization, Department of Reproductive Health and Research, Family and Community Health: Integrated management of pregnancy and childbirth. Pregnancy, childbirth, postpartum and newborn care: a guide for essential practice. Geneva, Switzerland; 2003.

14. World Health Organization, Department of Reproductive Health and Research: Coverage of maternity care: a listing of available information. 1996.

15. Koblinsky MA, Campbell $O$, Heichelheim J: Organizing delivery care: what works for safe motherhood? Bull World Health Organ 1999, 77:399-406.

16. De Brouwere V, Tonglet R, Van Lerberghe W: Strategies for reducing maternal mortality in developing countries: what can we learn from the history of the industrialized West? Trop Med Int Health 1998, 3:77|-782.

17. Adamu YM, Salihu HM: Barriers to the use of antenatal and obstetric care services in rural Kano, Nigeria. J Obstet Gynaecol 2002, 22:600-603.

18. Hodgkin D: Household characteristics affecting where mothers deliver in rural Kenya. Health Econ 1996, 5:333-340.

19. Mwaniki PK, Kabiru EW, Mbugua GG: Utilisation of antenatal and maternity services by mothers seeking child welfare services in Mbeere District, Eastern Province, Kenya. East Afr Med J 2002, 79: $184-187$

20. Smith JB, Coleman NA, Fortney JA, Johnson JD, Blumhagen DW, Grey TW: The impact of traditional birth attendant training on delivery complications in Ghana. Health Policy Plan 2000, 15:326-33|.

21. Ray AM, Salihu HM: The impact of maternal mortality interventions using traditional birth attendants and village midwives. J Obstet Gynaecol 2004, 24:5-I I.

22. Sibley LM, Sipe TA, Koblinsky M: Does traditional birth attendant training increase use of antenatal care? A review of the evidence. J Midwifery Womens Health 2004, 49:298-305.

23. United Nations: The Millennium Development Goals Report 2005. New York, USA; 2006.

24. Lindblade KA, Eisele TP, Gimnig JE, Alaii JA, Odhiambo F, ter Kuile FO, Hawley WA, Wannemuehler KA, Phillips-Howard PA, Rosen DH, Terlouw DJ, Adazu K, Vulule JM, Slutsker L: Sustainability of reductions in malaria transmission and infant mortality in western Kenya with use of insecticide-treated bednets: 4 to 6 years of follow-up. JAMA 2004, 29 I:257I-2580.

25. Phillips-Howard PA, Nahlen BL, Alaii JA, ter Kuile FO, Gimnig JE, Terlouw DJ, Kachur SP, Hightower AW, Lal AA, Schoute E, Oloo AJ, Hawley WA: The efficacy of permethrin-treated bed nets on child mortality and morbidity in western Kenya I. Development of infrastructure and description of study site. Am J Trop Med Hyg 2003, 68:3-9.

26. Adazu K, Lindblade KA, Rosen DH, Odhiambo F, Ofware P, Kwach J, van Eijk AM, Decock KM, Amornkul P, Karanja D, Vulule JM, Slutsker $L$ : Health and demographic surveillance in rural western Kenya: A platform for evaluating interventions to reduce morbidity and mortality from infectious diseases. Am J Trop Med Hyg 2005, 73: I I5I-I I 58.

27. Filmer D, Pritchett L: Estimating wealth effects without expenditure data - or tears: an application to educational enrollments in states of India. Demography 200 I, 38( I): I I5- I32.

28. Ministry of Health, Department of Reproductive Health, Department of Malaria Control, JHPIEGO: Focused Antenatal Care. Nairobi, Kenya; 2002.

29. Central Bureau of Statistics, Ministry of H, O. R. C. Macro: Kenya Demographic and Health Survey 2003. Calverton, Maryland, USA; 2004.
30. Kambarami RA, Chirenje MZ, Rusakaniko S: Antenatal care patterns and factors associated with perinatal outcome in two rural districts in Zimbabwe. Cent Afr J Med 1999, 45:294-299.

31. Massawe SN, Urassa EN, Nystrom L, Lindmark G: Effectiveness of primary level antenatal care in decreasing anemia at term in Tanzania. Acta Obstet Gynecol Scand 1999, 78:573-579.

32. Hamel MJ, Odhacha A, Roberts JM, Deming MS: Malaria control in Bungoma District, Kenya: a survey of home treatment of children with fever, bednet use and attendance at antenatal clinics. Bull World Health Organ 200I, 79:1014-1023.

33. Ndyomugyenyi R, Neema S, Magnussen P: The use of formal and informal services for antenatal care and malaria treatment in rural Uganda. Health Policy Plan 1998, 13:94-102.

34. Jahn A, Dar IM, Shah U, Diesfeld HJ: Maternity care in rural Nepal: a health service analysis. Trop Med Int Health 2000, 5:657-665.

35. Weil $\mathrm{O}$, Fernandez $\mathrm{H}$ : Is safe motherhood an orphan initiative? Lancet 1999, 354:940-943.

36. Gloyd S, Floriano F, Seunda M, Chadreque MA, Nyangezi JM, Platas A: Impact of traditional birth attendant training in Mozambique: a controlled study. J Midwifery Womens Health 200I, 46:210-216

37. Safe Motherhood: Skilled care during childbirth. Policy brief. New York, USA; 2002.

38. Rosenfield A: The history of the Safe Motherhood Initiative. Int J Gynaecol Obstet 1997, 59(Suppl 2):S7-S9.
Publish with Bio Med Central and every scientist can read your work free of charge

"BioMed Central will be the most significant development for disseminating the results of biomedical research in our lifetime. "

Sir Paul Nurse, Cancer Research UK

Your research papers will be:

- available free of charge to the entire biomedical community

- peer reviewed and published immediately upon acceptance

- cited in PubMed and archived on PubMed Central

- yours - you keep the copyright

Submit your manuscript here:

http://www.biomedcentral.com/info/publishing_adv.asp
BioMedcentral 